\title{
Estrategia psicoeducativa para la prevención de violencia de género en el noviazgo
}

\section{Psychoeducational strategy for the prevention of gender violence in engagement}

\author{
Dayana Díaz-Falcón ${ }^{1}$ \\ E-mail: ddfalcon94@gmail.com \\ ORCID: https://orcid.org/0000-0001-8537-4025 \\ Amarys Aimee Hernández-Caro ${ }^{2}$ \\ E-mail: amaryshernandez10@gmail.com \\ ORCID: https://orcid.org/0000-0001-7886-275X
}

Recibido: 07/12/2020, Aceptado: 26/03/2021

\begin{abstract}
RESUMEN
La violencia de género es un comportamiento aprendido que tiene raíces culturales, basadas en estereotipos, roles e identidades de género arraigadas, que ocasionan desequilibrios de poder. A pesar de esfuerzos gubernamentales y de organizaciones que trabajan para erradicarla, la sociedad tolera esta violencia y la ve reflejada en prácticas cotidianas. En la Universidad de Cienfuegos y el preuniversitario Martín Dihigo se identificaron mitos que legitiman la violencia de género, así como la naturalización acrítica de comportamientos cotidianos que constituyen violencia de pareja. Partiendo de este escenario, se trazó un plan de acciones educativas dirigido a la población adolescente y joven. Este tiene como objetivo crear espacios de reflexión para contribuir a la prevención de la violencia de pareja durante el noviazgo. Además, se promueve la cultura jurídica en clave de género como herramienta para la igualdad y la no violencia. Entre los principales resultados destaca la sensibilización con la temática, el desmontaje de los mitos existentes sobre violencia en el noviazgo, la construcción de un concepto de violencia de género, el reconocimiento de los diferentes tipos de violencia y la identificación de la relación existente entre amor romántico y violencia de pareja.
\end{abstract}

Palabras clave: violencia de pareja, género, prevención, adolescentes, jóvenes.

\section{ABSTRACT}

Gender violence is a learned behavior that has cultural roots, based on ingrained gender stereotypes, roles and identities, which cause imbalances of power. Despite government efforts and organizations working to eradicate it, society toleratesthis violence and sees it reflected in daily practices. At the University of

\footnotetext{
${ }^{1}$ Universidad de Cienfuegos. Cuba.

2 Universidad de Sancti Spíritus "José Marti". Cuba.
} 


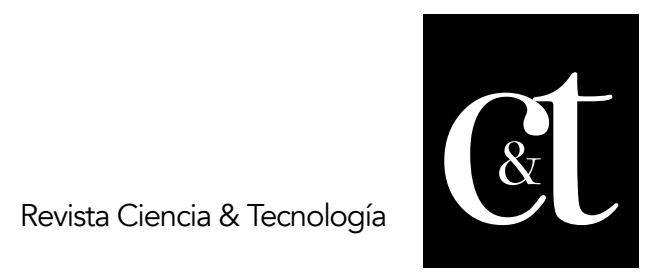

No. 30, 30 de abril de 2021

ISSN impreso: 1390 - 6321

ISSN online: 2661 - 6734

Cienfuegos and the Martín Dihigo High School, myths were identified that legitimize gender vilence, as weel as the uncritical naturalization of daily behaviors that constitute intimate partner violence. Starting from this scenario, an educational action plan was drawn up aimed at the adolescent and Young population. This aims to create spaces for reflection to contribute to the prevention of intimate partner violence during dating. In addition, the legal culture of gender issues is promoted as a tool for equality and non-violence. Among the main results, it is worth highlighting the awereness of the issue, the dismantling of existing myths about dating violence, the construction of a concept of gender violence, the recognition of the different types of violence and the identification of the relationship between love romantic and intimate partner violence.

Keywords: intimate partner violence, gender, prevention, adolescents, youth

\section{Introducción}

La Organización Mundial de la Salud (OMS) considera la violencia hacia la mujer como un problema de salud pública y de derechos humanos. Las estadísticas de la OMS reflejan que este tipo de violencia afecta a una amplia población mundial. Las cifras referidas pueden ser aún mayores debido a la falta de denuncias y a otros factores como la normalización social (Peña, 2011). A pesar de que históricamente no ha recibido la importancia que merece, la violencia de pareja durante el noviazgo, generalmente hacia la mujer, se ha configurado como uno de los problemas más graves de la sociedad actual. Como fenómeno universal este tipo de violencia se ha caracterizado por una clara invisibilidad en la que se minimizan sus efectos.

En este sentido, los distintos tipos de violencia hacia la mujer se normalizan a partir de las pautas de interacción social que han sido promovidas por la cultura machista. Puede darse en contextos tanto públicos como privados; como por ejemplo: en la familia, en la comunidad o en el ámbito laboral. Existen varios tipos de violencia, entre las que destacan la física, la psicológica y la sexual. La violencia física implica el empleo de fuerza contra el cuerpo de la víctima. La violencia psicológica se refiere a las maneras de tratar a mujer que limitan su libertad o niegan sus derechos y su dignidad. La violencia sexual se refiere desde los menosprecios a la sexualidad de la víctima, el acoso sexual hasta las violaciones (Alencar-Rodriguez y Cantera, 2013).

Las investigaciones internacionales sobre la violencia en la relación de pareja han colocado la mirada hacia la violencia contra la mujer, estas han registrado que existe una prevalencia entre un 60-69 \% solo entre $2-10 \%$ en el caso de mujeres hacia hombres (López-Angulo, Fundora-Quintero, Valladares-González, Ramos-Rangel y BlancoFleites, 2015). Según estadísticas de la OMS (2017), alrededor de una de cada tres mujeres en el mundo (35\%) han sufrido violencia física y/o sexual de pareja, afectando negativamente la salud física, mental, sexual y reproductiva de las mujeres. Las mujeres que son víctimas de violencia padecen de una variedad de problemas de salud y disminuye su capacidad para la participación pública y ciudadana.

Díaz, Hernández. Estrategia psicoeducativa para la prevención de violencia de género en el noviazgo 


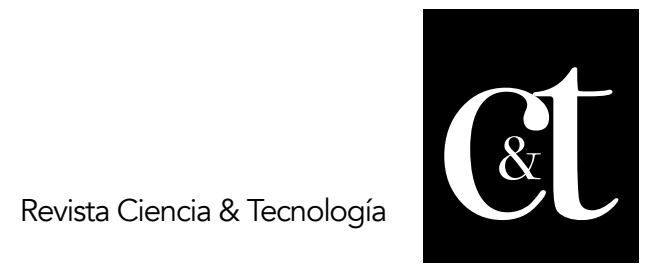

No. 30,30 de abril de 2021

ISSN impreso: 1390 - 6321

ISSN online: 2661 - 6734

La violencia hacia la mujer en la relación de pareja es entendida como toda acción activa o pasiva, innecesaria e injusta pudiendo estar condicionada por la estructura relacional jerarquizada, por la dinámica intersubjetiva en la configuración del vínculo y por las particularidades personológicas de ambos miembros, que es llevada a cabo por el hombre contra su pareja heterosexual, o mujer a la que esté o haya estado unido, con la intención de causarle daños en su capacidad de funcionamiento, de adaptación y de integración biopsicosocial y de transgredir sus derechos humanos fundamentales (López-Angulo, 2011).

La OMS refiere que como grupo etario las mujeres entre 15 y 44 años de edad corren mayor riesgo de ser violadas o maltratadas. Una posible explicación a este hecho está directamente relacionada con la dificultad que tienen adolescentes y jóvenes para reconocer que son víctimas del maltrato (GarcíaDíaz, Fernández-Feito, Rodríguez-Díaz, López-González, Mosteiro, \& Lana, 2013; Vizcarra, Poo, \& Donoso, 2013). En cuanto a la prevalencia de violencia de pareja entre adolescentes, se ha reportado un $20 \%$ de violencia física y un $9 \%$ de violencia sexual. Las mujeres recurren más a amenazas e insultos y en ocasiones agresiones físicas, mientras que los hombres ejercen más la violencia sexual (Shorey, Fite, Cohen, Stuart \& Temple, 2018; Wincentak et al., 2017 citados en Rozo-Sánchez, Moreno-Méndez, Perdomo-Escobar y Avendaño-Prieto, 2019).

Investigar este tipo de violencia en parejas jóvenes, para intentar erradicarla, se torna una tarea imprescindible ya que en esta etapa de especial vulnerabilidad, transición y cambios, los comportamientos violentos pueden instaurarse de forma gradual en función del compromiso que vayan adquiriendo los miembros de la pareja (Hernando, 2007). Se puede convertir un claro precursor de la violencia de género en la etapa adulta y, con ello, en un serio problema de salud pública (Muñoz-Rivas et al., 2010).

En relación al diagnóstico de esta problemática, las investigaciones se centran en estudiar estas conductas agresivas y su relación con determinadas variables como el sexo, clima familiar, identificar factores que obstaculizan el tratamiento de este fenómeno, así como en especificar el tipo de violencia más frecuente (Pazos-Gómez, Oliva-Delgado y Hernando-Gómez, 2014; López-Angulo et al., 2015). Los principales resultados de estos estudios refieren que se deben tener en cuenta algunos factores de riesgo como la disfuncionalidad familiar y el ser víctimas de violencia durante la infancia. De igual forma se refiere una prevalencia de la violencia psicológica y física en las relaciones de pareja durante el noviazgo.

El desarrollo de las relaciones de pareja es un proceso complejo, donde muchos adolescentes consideran la agresión dentro de una relación de pareja como algo normal (Muñoz-Rivas, Graña, O'Leary y González, 2007). Esta normalización conlleva a la justificación de los actos agresivos percibiéndolos como algo aceptable y a la incorporación de estas conductas en sus estilos interactivos habituales, siendo una forma de resolver conflictos en muchas parejas. Por otro lado, son varios los estudios que indican que las relaciones en esta etapa adquieren un carácter recíproco o bidireccional, es decir, ambos miembros de la pareja ejercen y sufren conductas abusivas, fundamentalmente de tipo físico y psicológico (Rubio-Garay, López-González, Saúl y Sánchez-Elvira, 2012; Samaniego y Freixas, 2010). 


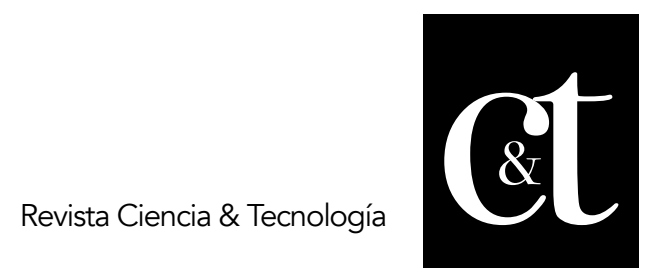

No. 30, 30 de abril de 2021

ISSN impreso: 1390 - 6321

ISSN online: 2661 - 6734

También es preciso tener en cuenta que uno de los aspectos que aumenta la invisibilización de la violencia durante el noviazgo es la idealización que adolescentes y jóvenes realizan de las conductas violentas, con base en el "amor romántico", y la justificación y el hecho de quitarle importancia a comportamientos violentos como son los celos, el control obsesivo, etcétera.

Ello refuerza la necesidad y la pertinencia de desarrollar nuevos estudios en esta área. Cuba no es ajena a esa realidad, por lo que se ha venido desarrollando un arduo trabajo por la Federación de Mujeres Cubanas, el CENESEX, el Centro Oscar Arnulfo Romero y la Cátedra de la Mujer. Cada año se llevan a cabo las Jornadas Cubanas por la No Violencia contra las Mujeres y las Niñas, se desarrolló la campaña Eres Más y actualmente la campaña Evoluciona, la campaña Únete Cuba bajo el mensaje Yo digo No y en La Habana la campaña Súmate. A pesar de ello, el Anuario Estadístico de Salud refleja que las cifras de muertes por agresiones cobran más vidas que el SIDA y se ubican en la lista de las primeras 35 causas de muerte en el país (Moraga, 2017).

La eliminación de la violencia contra la mujer es condición indispensable para su desarrollo y para su plena participación en todas las esferas de vida, en igualdad de condiciones con el hombre, en todos los campos. Las ciencias sociales y humanas tienen un papel fundamental en la comprensión, prevención e intervención de esta problemática a fin de erradicarla, como propone el tercer objetivo del milenio. Desde este escenario, en el marco de la campaña por la No Violencia Hacia las Mujeres y las Niñas, se diseñó e implementó un plan de acciones educativas con carácter preventivo dirigido a los adolescentes y jóvenes. Su objetivo es crear espacios de reflexión para contribuir a la prevención de la violencia de pareja durante el noviazgo.

\section{Metodología}

El estudio se llevó a cabo por especialistas del Gabinete Psicopedagógico de la Universidad de Cienfuegos, con adolescentes y jóvenes del Municipio de Cienfuegos. El trabajo se llevó a cabo en 3 etapas. La primera estuvo dirigida al diagnóstico de la problemática. En la segunda etapa se diseñó un plan de acciones educativas dirigido a la población adolescente y joven. En la tercera etapa se implementó y evaluó el plan de acciones.

\section{Primera Etapa: Diagnóstico de la problemática}

Para llevar a cabo el diagnóstico se utilizó la observación participante en las dos sedes de la Universidad de Cienfuegos (UCf) y en el IPU Martín Dihigo con el objetivo de identificar comportamientos de violencia de pareja. Además, se entrevistaron a estudiantes de ambas instituciones y a otros informantes claves.

\section{Segunda etapa: Diseño del plan de acciones educativas}

Los especialistas del Gabinete Psicopedagógico de la UCf trazaron un plan de acciones educativas dirigido a la población adolescente y joven. Este tiene como objetivo crear espacios de reflexión para contribuir a la prevención de la violencia de pareja durante el noviazgo.

Las acciones abarcaron tres áreas: debates en redes sociales, exposiciones del tema en las dos sedes de la UCf y cuatro talleres. 


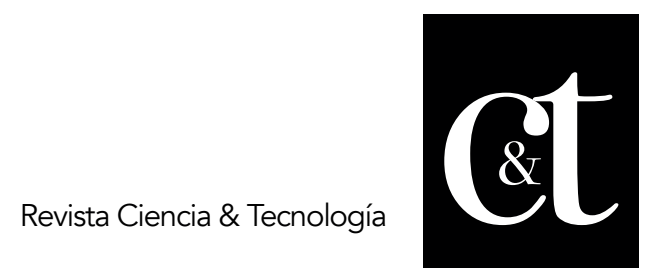

No. 30, 30 de abril de 2021

ISSN impreso: 1390 - 6321

ISSN online: 2661 - 6734

Los talleres tienen la intención de profundizar en la temática e introducir metodologías de intervención con adolescentes y jóvenes. Se emplea un lenguaje basado en lo cotidiano que permita la identificación y comprensión por parte de la juventud. Para un mayor acercamiento, sensibilización y toma de conciencia a la problemática social de la violencia de pareja se utilizan ejemplos y anécdotas.

Los talleres van dirigidos a desmontar los mitos existentes sobre la violencia hacia la mujer, construir un concepto sobre violencia de género y violencia de pareja, reconocer los diferentes tipos de violencia, identificar la relación existente entre amor romántico y violencia de pareja y promover la cultura jurídica en clave de género como una herramienta para la igualdad y la no violencia contra las mujeres. El sistema de talleres es el siguiente:

\section{Taller 1: Realidades y mitos}

Objetivos:

- Determinar el nivel de conocimiento de los estudiantes con respecto a la problemática de la violencia de pareja.

- Desmontar mitos sobre la violencia hacia la mujer.

Inicio:

Al comienzo del taller se lleva a cabo el caldeamiento del grupo mediante la técnica "Presentación por pareja". Esta tiene como objetivos tratar de romper bloqueos e inhibiciones iniciales, generar un ambiente más cercano entre los participantes y percibir sus expectativas sobre el taller. Se les pide a los participantes que escojan una pareja que no conozcan o que conozcan menos. Una vez se han formado las parejas, se les dice que disponen de tres minutos para intercambiar información sobre sus nombres, lugar de vivienda y que estudia.

Una vez se terminen los tres minutos asignados, se les propone el cambio de parejas para que, con la segunda pareja, durante otros tres minutos, se cuenten: quién las invitó a este taller, por qué aceptó la invitación y qué cosas tuvo que dejar pendientes para poder llegar al taller. Luego, escogiendo a una tercera pareja, se dirán: qué quisieran aprender de este taller y para qué creen que les puede servir. Por último, con todos los participantes reunidos en un solo grupo, algunos de ellos dirán con quiénes se encontraron, sus nombres, sus historias y sus expectativas acerca del taller; lo que se diga se dejará escrito en carteleras para tenerlo presente durante el trabajo y para aclararlo, como punto de partida. Finalizando el ejercicio para romper el hielo, se presentan los objetivos y temas del taller.

\section{Desarrollo}

La técnica central se denomina "Realidades y mitos" (Martínez, 2012). El objetivo es analizar frases para identificar los roles y estereotipos. Se entregan varias tarjetas con enunciados a los participantes. Luego de breves minutos se selecciona, a un estudiante para que lea en voz alta el contenido de su tarjeta y refiera si lo considera un mito o una realidad, justificando su respuesta. Las preguntas para estimular el debate son las siguientes: ¿Cree que es cierto o falso lo que dice la tarjeta? 


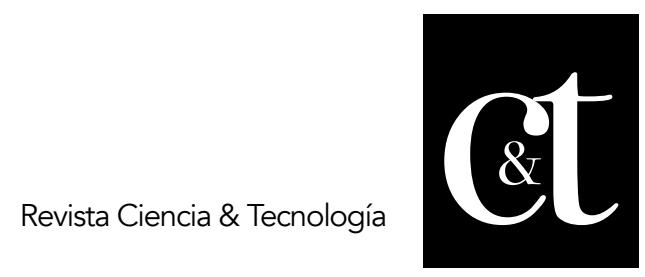

No. 30, 30 de abril de 2021

ISSN impreso: 1390 - 6321

ISSN online: 2661 - 6734

Por otro lado, ¿qué idea sustenta o qué quiere decir? Al finalizar cada estudiante, el resto del grupo debe argumentar su desacuerdo o no con lo planteado. Las preguntas para estimular el debate son las siguientes: ¿Por qué o para qué utilizan las personas esta frase?, ¿Beneficia o perjudica a alguien? En cada intervención el coordinador debe ofrecer una explicación respecto al mito o la realidad, aportando información nueva y datos interesantes.

\section{Cierre:}

El taller cierra con la técnica "La frase del día". Esta tiene como objetivos consolidar y evaluar los conocimientos adquiridos y evaluar le eficacia del taller. Para ello se les pide a los participantes que digan en una frase lo que ha resultado más significativo para ellos durante el taller.

\section{Taller 2: Tipos de violencia}

Objetivos:

- Construir un concepto sobre violencia de género y violencia de pareja.

- Reconocer los diferentes tipos de violencia.

Inicio:

Al comienzo del taller se lleva a cabo la técnica de activación "el detalle que falta". Esta tiene como objetivos motivar a los participantes para el comienzo del taller. Se forman parejas de manera voluntaria. Una pareja a la vez pasa al centro, luego de observarse con mucha atención, cambian 5 detalles de su apariencia. Se colocan nuevamente frente a frente y tratan de adivinar los cambios, el grupo mediante pistas ayuda a adivinar los cambios que falten.

Desarrollo:

Se emplea la técnica "Tormenta de ideas" con el objetivo de construir un concepto sobre violencia de género y pareja. Se solicita a los participantes que hablen libremente, sin ningún tipo de cortapisas, vallan diciendo lo primero que se les ocurre sobre la violencia de género y de pareja, aunque parezca absurdo. El coordinador, apunta todas las ideas en la pizarra. En un segundo momento, se ordena todo lo aportado y se analiza entre todos los participantes conformando el concepto de violencia de género y violencia de pareja.

En una segunda fase se lleva a cabo la técnica "Juego de roles" con el objetivo de identificar los diferentes tipos de violencia. Para ello se conforman subgrupos, a cada uno se le asigna un tipo de violencia hacia la mujer. A partir del tipo de violencia asignado cada sub-grupo debe conformar una historia, distribuir los personajes entre los miembros del sub-grupo y dramatizar la historia sin decir qué tipo de violencia están representando. El resto del grupo debe identificar el tipo de violencia al final de cada representación. Por último, con todos los participantes reunidos en un solo grupo se debate sobre las historias dramatizadas, los tipos de violencia que se apreciaron, otros existentes, los desenlaces de las historias, etc.

Cierre:

El taller cierra con la técnica "El gesto". Esta tiene como objetivos identificar con un gesto cómo se sintieron o lo aprendido durante la sesión e identificar el

49

Díaz, Hernández. Estrategia psicoeducativa para la prevención de violencia de género en el noviazgo 
cumplimiento de los objetivos. Para ello se le pide a cada participante que exprese con un gesto como se sintió o lo que aprendió durante el taller.

Taller 3: Amor romántico y violencia de pareja.

Objetivos:

- Desmontar creencias erróneas sobre el amor romántico.

- Identificar la relación existente entre amor romántico y violencia de pareja.

Inicio:

Se comienza el taller con una pregunta sencilla: ¿Qué es el amor? Se realizan varias preguntas para estimular el debate. Se recogen los criterios de los participantes en un papelógrafo. Luego se identifica la presencia de creencias erróneas sobre el amor romántico, las cuales son devueltas a los participantes por el coordinador mediante el análisis de los 4 grupos de mitos sobre el amor romántico (Figura 1).

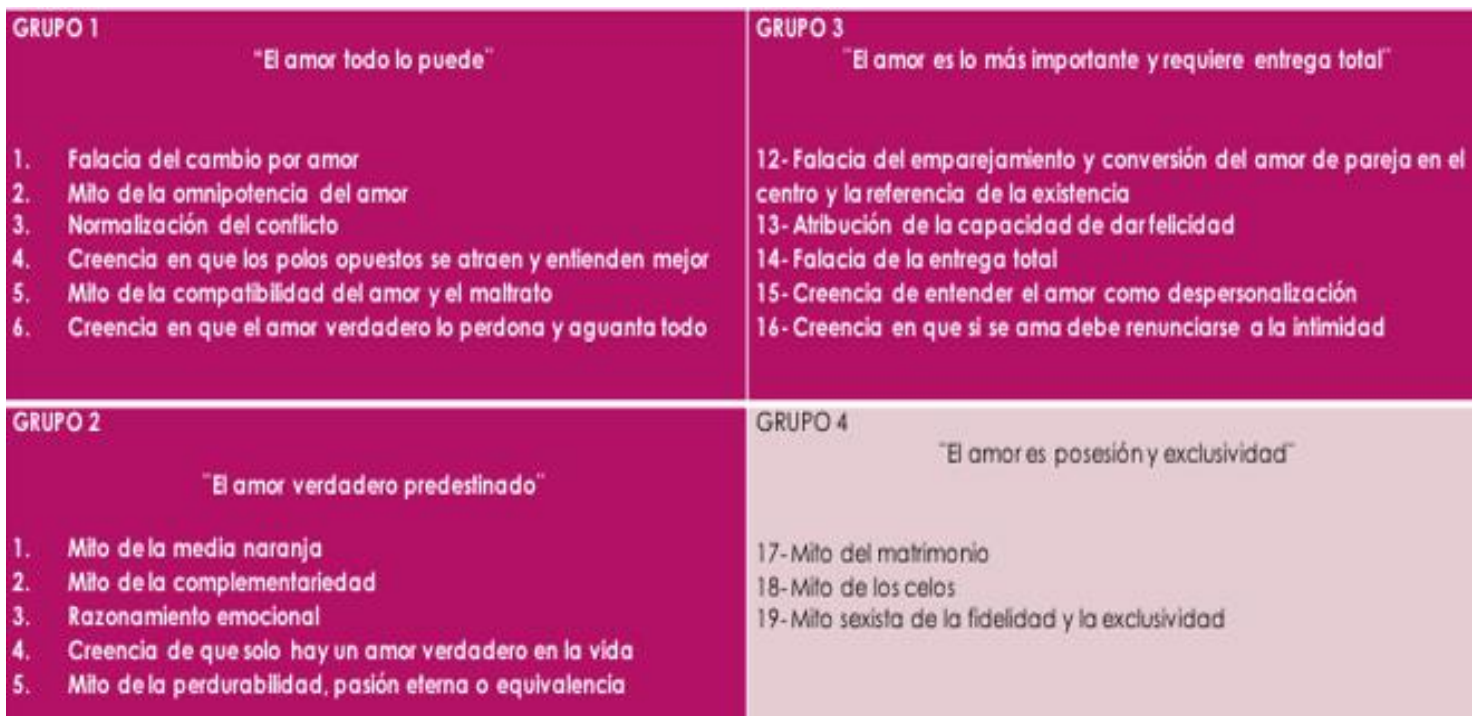

Figura 1. Mitos más comunes del amor romántico Fuente: elaboración propia

\section{Desarrollo}

Se comparten con los estudiantes experiencias reales de mujeres víctimas de violencia en la relación de pareja. Estas experiencias son tomadas del libro "No te mueras por mí" (Imízcoz, 2015), el cual comparte lo ocurrido a 25 mujeres. Se tiene como objetivo sensibilizar con la problemática e identificar la relación existente entre los mitos del amor romántico y la violencia de pareja.

Cierre:

El cierre del taller se lleva a cabo mediante la técnica "¿Cómo llegué y cómo me voy?". 


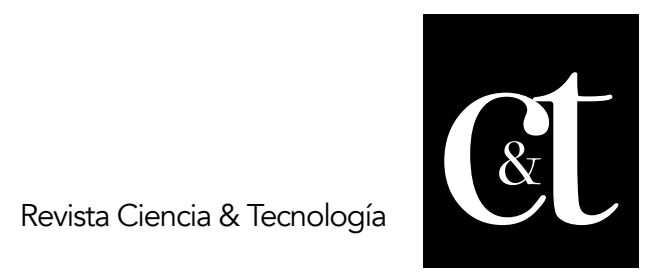

No. 30, 30 de abril de 2021

ISSN impreso: 1390 - 6321

ISSN online: 2661 - 6734

Esta tiene como objetivos identificar las impresiones de los participantes ante y después del taller y evaluar el cumplimiento de los objetivos. Para ello se le pide a cada participante que exprese en breves palabras como se sintió al principio y al final del taller.

Taller 4: Cultura jurídica en clave de género.

Objetivo:

- Promover la cultura jurídica en clave de género como una herramienta para la igualdad y la no violencia contra las mujeres.

La técnica empleada en este taller es la charla educativa. Para desarrollar la charla se invita a especialistas del Gabinete de Orientación Jurídica. Una vez terminada la exposición de los especialistas se inicia un debate con los participantes.

\section{Tercera etapa: Puesta en marcha del plan de acciones.}

Para generar debate en las redes sociales se compartieron videos, memes, fotografías y comentarios sobre el tema en grupos y páginas de la UCf; invitando a los jóvenes a participar y debatir sobre el tema.

Las exposiciones del tema realizadas en ambas sedes de la UCf integraban juegos participativos, debates e intercambio de experiencias. Además, contaron con la presentación de libros y revistas, la elaboración de carteles y dibujos que expresaran las experiencias, conocimientos y sentimientos de los participantes sobre la violencia de pareja. Los juegos de participación incluían ordenar los mitos y las realidades sobre el tema y responder preguntas polémicas. En todo momento se buscaba generar debate y reflexión, así como desmontar creencias erróneas y visibilizar la violencia machista.

Se desarrollaron los cuatro talleres en la UCf sede "Conrado Benítez", con estudiantes de preuniversitario, estudiantes de la residencia estudiantil y estudiantes de la Facultad de Educación. Además, los dos primeros talleres se desarrollaron el IPU Martín Dihigo (Tabla 1).

Tabla 1. Población y muestra

\begin{tabular}{|l|c|}
\hline \multicolumn{1}{|c|}{ Estudiantes } & Frecuencia \\
\hline $\begin{array}{l}\text { Estudiantes del colegio universitario } \\
\text { de la UCf }\end{array}$ & 9 \\
\hline $\begin{array}{l}\text { Estudiantes de la Residencia } \\
\text { estudiantil de la Sede "Conrado } \\
\text { Benítez" }\end{array}$ & 12 \\
\hline $\begin{array}{l}\text { Estudiantes de la Facultad de } \\
\text { Educación }\end{array}$ & 18 \\
\hline Estudiantes del IPU & \\
\hline
\end{tabular}

Fuente: elaboración propia

Díaz, Hernández. Estrategia psicoeducativa para la prevención de violencia de género en el noviazgo 


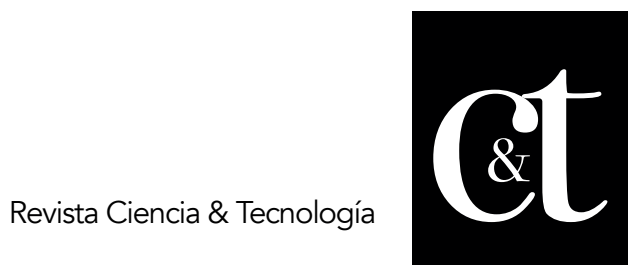

No. 30, 30 de abril de 2021

ISSN impreso: 1390 - 6321

ISSN online: 2661 - 6734

\section{Resultados y discusión}

En la etapa de diagnóstico se pudo constatar la existencia de mitos que legitiman la violencia de género en la UCf y el preuniversitario, así como la naturalización acrítica de comportamientos cotidianos que constituyen violencia de pareja, fundamentalmente hacia la mujer. Entre ellos: "entre marido y mujer nadie se debe meter", "los problemas de las parejas son asuntos privados", "el amor lo puede y aguanta todo", "la violencia se da cuando las personas son agresivas o delincuentes", "las violaciones ocurren en lugares oscuros y solitarios", "las violaciones son por desconocidos en lugares peligrosos", "hay mujeres que les gusta el golpe y no dejan a sus parejas", "hay veces que se lo buscan", "son piropos que ayudan a la mejor autoestima", entre otros.

En la figura 2 se aprecia que el $70 \%$ de las personas entrevistadas consideran la violencia de pareja como un asunto privado y el $75 \%$ le confieren culpabilidad a la víctima por la violencia a que son sometidas. Mientras un $50 \%$ de los entrevistados tienen creencias erróneas sobre la omnipotencia del amor y la compatibilidad entre amor y maltrato y un $40 \%$ consideran los "piropos" como halagos que favorecen el aumento de la autoestima. También emergieron mitos sobre las personas que ejercen la violencia, así como los lugares donde ocurren las violaciones y quienes la llevan a cabo, por un 30 y $25 \%$ de los entrevistados respectivamente.

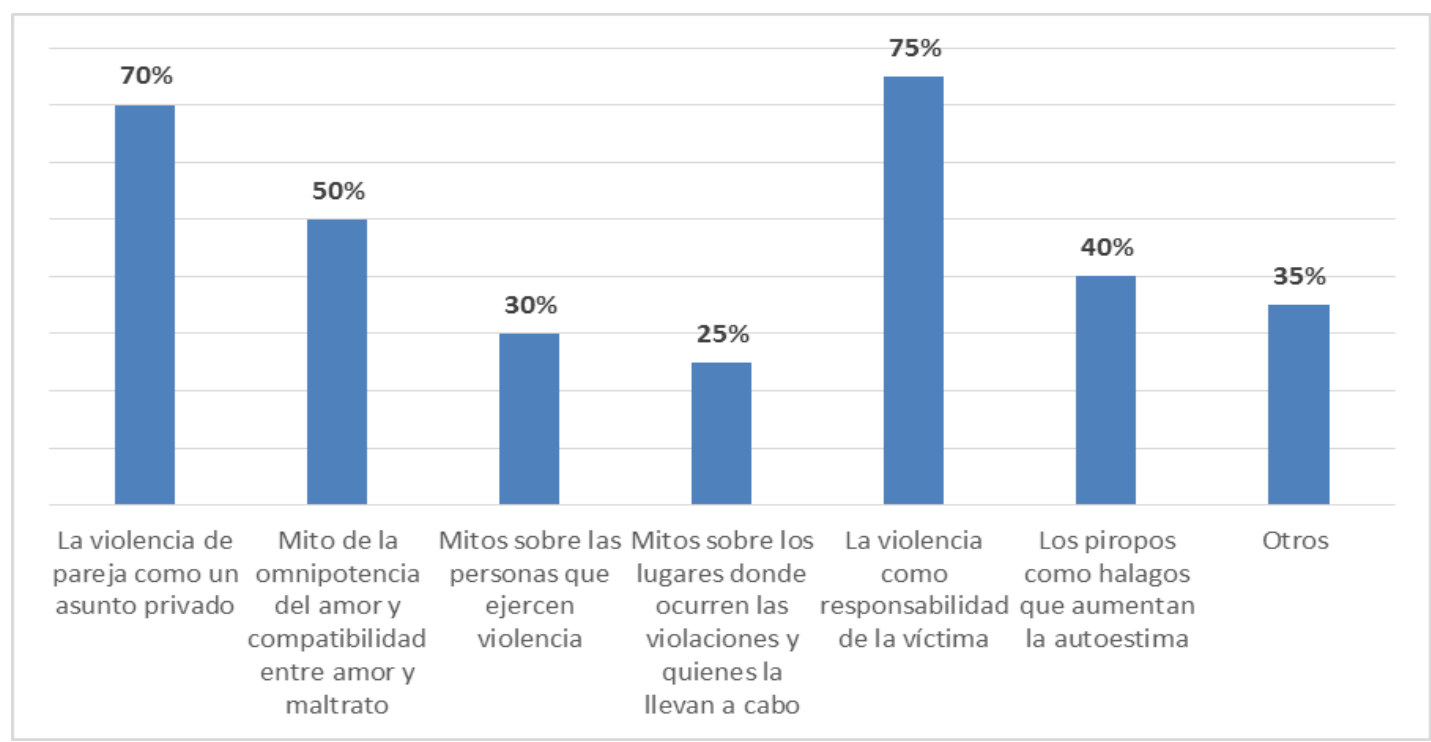

Figura 2. Mitos que legitiman la violencia de género (resultados del diagnóstico) Fuente: elaboración propia

En el taller 1 se logró el caldeamiento del grupo, rompiendo bloqueos e inhibiciones para comenzar a trabajar con el tema. Los estudiantes interactuaron creando un ambiente más cercano al intercambiar información personal. Entre los motivos para asistir al taller se encontraron: "invitación de un amigo", "curiosidad", "interés en el tema", motivación a partir de la propaganda de redes sociales y el conocimiento adquirido en las exposiciones. 


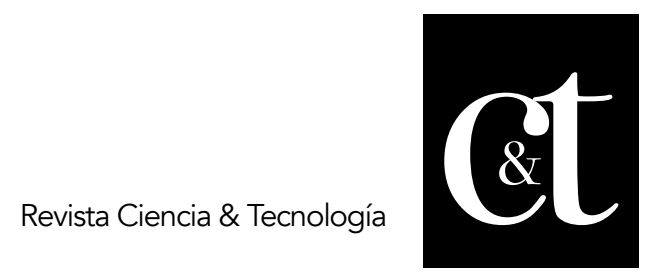

No. 30,30 de abril de 2021

ISSN impreso: 1390 - 6321

ISSN online: 2661 - 6734

Se clarificaron las expectativas de los estudiantes sobre el taller. Los mitos que predominaron fueron los siguientes: "los problemas de las parejas son asuntos privados", "las mujeres agredidas en una relación de pareja son masoquistas", "los agresores están enfermos, son pobres o alcohólicos", "la violencia produce respeto" y "el amor lo puede y aguanta todo". Debido a ello se llevaron a cabo reflexiones sobre quienes ejercen la violencia, el por qué las mujeres no denuncian la agresión y algunos factores de riesgo y protectores. Se comprobó el cumplimiento de los objetivos del taller mediante la técnica de cierre. Aunque se aprecia la necesidad de seguir trabajando en el desmontaje de los mitos que legitiman la violencia de pareja.

En el taller 2, luego de motivar a los estudiantes para el comienzo del taller, se realiza la técnica central dirigida a construir un concepto sobre violencia de género y violencia de pareja. Entre las ideas que emergieron destacan: "maltratar", "golpear", "atacar por el género", "maltratar a tu pareja", "cuando hay una violación", "hacer daño". Se aprecia la identificación del concepto con la violencia física, desentendiendo otros tipos de violencia.

En un segundo momento los estudiantes fueron capaces de reconocer la existencia de otros tipos de violencia, presentes también en las relaciones de pareja durante el noviazgo. En este sentido, emergieron en el debate varias experiencias de personas cercanas a ellos. Pudiendo identificar la violencia física, sexual, verbal, económica y psicológica. Se reconoce, además, que tanto los hombres como las mujeres pueden ser víctimas y/o agresores pero que son las mujeres quienes más la padecen. De esta manera pudieron construir un concepto propio sobre violencia de género y violencia de pareja. Los gestos que realizaron los estudiantes para el cierre demostraron su bienestar con el taller.

En el taller 3 se evidencia que los estudiantes se sintieron identificados con varios comportamientos violentos que se enmascaran debido a las creencias erróneas sobre el amor romántico. En este sentido, señalaron que "habían vivenciado relaciones posesivas por creer que los celos eran muestra de amor", que "varias personas no permiten que su pareja tenga privacidad" y "le controlan constantemente el teléfono", entre otros comportamientos. Las historias de mujeres violentadas por sus parejas lograron sensibilizar a los estudiantes sobre la violencia de pareja, fundamentalmente hacia las mujeres. Los estudiantes expresaron que los protagonistas de las historias "parecían muy enamorados", "no sabían cómo pudieron hacer eso", "no hay nada que justifique ese comportamiento tan violento", "por el mensaje parecía una persona muy enamorada que no sería capaz de hacerle daño", etc. En este momento se les explicó a los estudiantes el ciclo de la violencia.

De manera general, en el transcurso del taller y por la técnica de cierre se puso apreciar que los estudiantes fueron capaces de identificar la relación existente entre los mitos del amor romántico y la violencia de pareja. Además, se lograron desmontar creencias erróneas sobre el amor romántico.

En el taller 4 los especialistas del Gabinete de Orientación Jurídica desarrollaron una charla centrada en presentar la cultura jurídica en clave de género como una herramienta para la igualdad y la no violencia contra las mujeres.

Díaz, Hernández. Estrategia psicoeducativa para la prevención de violencia de género en el noviazgo 


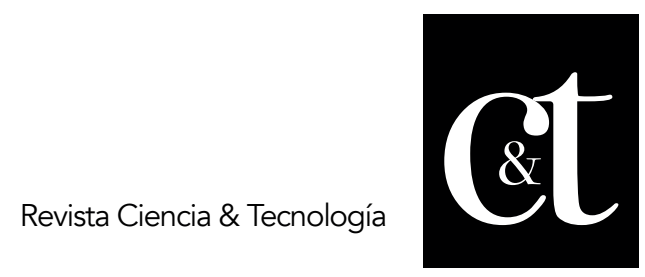

No. 30, 30 de abril de 2021

ISSN impreso: 1390 - 6321

ISSN online: 2661 - 6734

Abordaron varios aspectos, como son: los motivos por lo que las mujeres no denuncian, ejemplos de frases negativas por parte de los servidores públicos que disuaden a la mujer de denunciar la violencia, algunas consecuencias de la violencia contra la mujer y cómo acceder a servicios legales. En todo momento se incentivó el debate con los estudiantes.

Varias investigaciones sostienen como los estereotipos de género son un factor de riesgo para la violencia de pareja en el noviazgo (Bonilla, Rivas y Vázquez, 2017; López, 2019; Rozo-Sánchez, Moreno-Méndez, Perdomo-Escobar y Avendaño-Prieto, 2019). Rozo-Sánchez y colaboradores (2019) desarrollaron dos modelos de violencia de pareja en adolescentes colombianos que fueron determinados diferencialmente por el sexo, relacionándose los problemas de adaptación con la agresión en el caso de los hombres y con la victimización en las mujeres. Por su parte, un estudio desarrollado con 423 personas entre 17 y 81 años evidenció la existencia de creencias erróneas sobre quienes padecen o cometen la violencia (Ferrer-Pérez, López-Prats, Bosch-Fiol y Navarro-Guzmán, 2016).

En correspondencia, el presente estudio constató la existencia de mitos sobre violencia de género y violencia de pareja que ponen a las mujeres en una situación de vulnerabilidad social.

López (2019) plantea que las adolescentes conocen el concepto de violencia de pareja debido a la frecuencia con la que es tratado, mediante charlas, campañas y por los medios de comunicación; aunque no saben identificar actitudes de desigualdad por parte de su pareja. En correspondencia en el presente estudio se constató la dificultad para detectar cuando son víctimas de violencia en la relación de noviazgo. Sin embargo, se aprecia la necesidad de trabajar más el tema debido a que existen déficit en la concepción de violencia que dominan los adolescentes; ignorando tipos de violencia diferentes a la física.

En esta línea, una investigación que empleó grupos de discusión con adolescentes entre 15 y 19 años y un estudio de caso de una adolescente de 15 años de edad, arrojó resultados similares. Se aprecia ausencia de información sobre el tema de violencia de género y violencia de pareja, dificultad para identificar los comienzos de violencia en una relación tóxica, acompañada de distorsión en las interpretaciones hostiles y consecuencias negativas en la víctima (Romero, Montilla y Martín, 2017). Todos estos elementos destacan la necesidad de llevar a cabo acciones de prevención de la violencia de pareja.

En un estudio desarrollado en un centro penitenciario, con una muestra de 30 hombres agresores y 30 mujeres víctima de violencia de género, se aprecian mayores sesgos cognitivos en caso de los hombres en relación a anteponer necesidades, deseos e intereses propios frente a los de la pareja y a la anticipación del pensamiento necesario en el amor romántico. En el caso de las mujeres destaca la necesidad de celotipia y atracción única hacia la pareja para describir el amor auténtico (Picado, Yurrebaso, Álvarez y Martín, 2019). Los autores del estudio plantean la necesidad de analizar la definición de amor romántico por parte de hombres y mujeres, para detectar las distorsiones cognitivas más relacionadas con la violencia de género. 


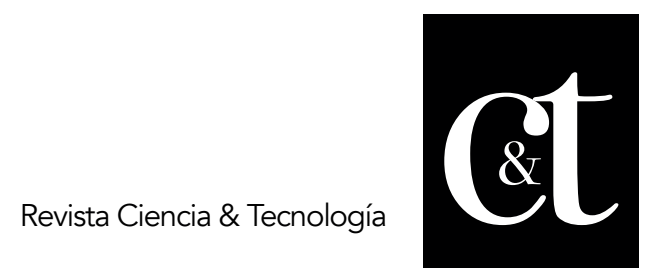

No. 30, 30 de abril de 2021

ISSN impreso: 1390 - 6321

ISSN online: 2661 - 6734

Cuestión que se trabajó con los adolescentes y jóvenes, donde se evidenciaron la existencia de varios mitos sobre el amor romántico tanto en los talleres como en el intercambio llevado a cabo durante las exposiciones.

Bonilla y colaboradores (2017) encontraron que los mecanismos de control y abuso de intimidad por parte de la pareja a través de las TIC se encuentran muy aceptados y se producen bidireccionalmente entre mujeres y hombres. Cuestión que fue también identificada por los estudiantes que participaron en los talleres.

\section{Conclusiones}

Se pudo apreciar la sensibilización con la problemática por parte de los estudiantes. El desmontaje de alguno de los mitos sobre la violencia de género y más específicamente la violencia de pareja y el amor romántico. Aunque, se requiere seguir trabajando en este sentido ya que todavía se aprecia la existencia de mitos.

Los estudiantes fueron capaces de construir un concepto sobre violencia de género y violencia de pareja, a partir de la diferenciación de conceptos como sexo, género, estereotipos de género, entre otros. Aunque, venían con la idea de violencia solo como agresión física. Identificaron diferentes tipos de violencia de género, las cuales aprecian en sus vidas cotidianas. Además, encontraron la relación entre amor romántico y violencia de pareja, a pesar de que continúan teniendo creencias erróneas con respecto al amor romántico y el ideal de pareja.

El plan de acciones educativas contribuyó a visibilizar el tema de la violencia de pareja en el noviazgo y a sensibilizar con la problemática. Además, aumentó el conocimiento de los estudiantes preuniversitarios y universitarios sobre estos temas, contribuyendo a la prevención de este tipo de violencia. Es importante insistir en la necesidad de la prevención de la violencia de pareja, comenzando por la educación en la igualdad. Los centros educativos tienen un lugar relevante en educar para establecer relaciones de parejas sanas y prevenir la violencia.

\section{Referencias}

Alencar-Rodríguez, R. y Cantera, L. M. (2013). Intervención en violencia de género en la pareja: el papel de los recursos institucionales. Athenea Digital, 13(3), 75-100.

Bonilla, E., Rivas, E. y Vázquez, J. J. (2017). Tolerancia y justificación de la violencia en relaciones de pareja adolescentes. Apuntes de Psicología, 35(1), 55-61.

Ferrer-Pérez, V. A., López-Prats, L., Bosch-Fiol, E. y Navarro-Guzmán, C. (2016). La vigencia de los mitos sobre la violencia contra las mujeres en la pareja. Información Psicológica, 111, 2-17.

García-Díaz, V., Fernández-Feito, A., Rodríguez-Díaz, F.G., López-González, M.L., Mosteiro, M.D. y Lana, A. (2013). Violencia de género en estudiantes de enfermería durante sus relaciones de noviazgo. Atención Primaria, 45, 290-296.

Hernando, A. (2007). La prevención de la violencia de género en adolescentes. Una experiencia en el ámbito educativo. Apuntes de Psicología, 25(3), 325340.

Imízcoz, J. (2015). No te mueras por mí. Editorial Vida Mujer.

Díaz, Hernández. Estrategia psicoeducativa para la prevención de violencia de género en el noviazgo 


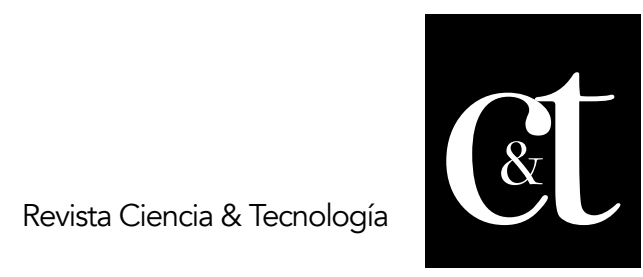

No. 30, 30 de abril de 2021

ISSN impreso: 1390 - 6321

ISSN online: 2661 - 6734

López-Angulo, L. (2011). Violencia hacia la mujer por su pareja. Intervenciones orientadas a su maneja integral.

López-Angulo, L., Fundora-Quintero, Y., Valladares-González, A., Ramos-Rangel, Y. y Blanco-Fleites, Y. (2015). Prevalencia de la violencia física y psicológica en relaciones de pareja heterosexuales. Revista Finlay, 5(3).

López, L. (2019). Violencia de género en parejas adolescentes (Tesis de Diploma). Universitat Juame.

Martínez, B. (2012). Guía de taller de prevención de la violencia contra las mujeres. Dirección General de Prevención del Delito y Participación Ciudadana, SSP. 